\title{
Norois
}

Environnement, aménagement, société

206 | 2008/1

Itinéraires de lieux touristiques littoraux

\section{Un SIG pour connaître et pour gérer la plaisance dans le Finistère}

A GIS dedicated to boating in the Finistère (France)

Guillaume Nardin, Iwan Le Berre et Louis Brigand

\section{OpenEdition}

Journals

Édition électronique

URL : http://journals.openedition.org/norois/270

DOI : $10.4000 /$ norois. 270

ISBN : 978-2-7535-1554-3

ISSN : $1760-8546$

Éditeur

Presses universitaires de Rennes

Édition imprimée

Date de publication : 1 mars 2008

ISBN : 978-2-7535-0690-9

ISSN : 0029-182X

Référence électronique

Guillaume Nardin, Iwan Le Berre et Louis Brigand, « Un SIG pour connaître et pour gérer la plaisance dans le Finistère », Norois [En ligne], 206 | 2008/1, mis en ligne le 01 mars 2010, consulté le 30 avril 2019. URL : http://journals.openedition.org/norois/270 ; DOI : 10.4000/norois. 270

(c) Tous droits réservés 


\title{
UN SIG POUR CONNAîTRE ET POUR GÉRER LA PLAISANCE DANS LE FINISTÈRE
}

\author{
Guillaume Nardin ${ }^{1}$, Iwan Le Berre ${ }^{2}$, Louis Brigand ${ }^{2}$ \\ ${ }^{1}$ MASTER 2 EXPERTISE ET GESTION DE L'ENVIRONNEMENT LITTORAL \\ ${ }^{2}$ GÉOMER -LETG UMR 6554 CNRS \\ (Université de Bretagne occidentale), \\ Institut Universitaire Européen de la Mer \\ Technopôle Brest-Iroise, place Nicolas-Copernic - 29280 PlouzANÉ \\ guinardin@yahoo.fr,iwan.leberre@univ-brest.fr,louis.brigand@univ-brest.fr
}

\section{RÉSUMÉ}

En plus d'un réel atout de développement économique, la plaisance constitue un outil de promotion touristique pour le département du Finistère. Pourtant, cette activité qui s'est développée jusqu'à présent sans réel contrôle, ni organisation globale, nécessite une structuration en raison de son emprise spatiale croissante, de l'augmentation du nombre de pratiquants et de la pression qui en découle sur le littoral et sur ses différents usagers.

Nautisme En Finistère (NEF) joue un rôle fédérateur pour l'organisation et le développement de la plaisance dans le département et, à ce titre, a engagé plusieurs études complémentaires visant à améliorer la connaissance de cette activité et des infrastructures liées. Les données collectées lors de ces études ont été intégrées à un système d'information géographique qui fourni la base d'un observatoire de la plaisance dans le Finistère sur lequel doivent s'appuyer les stratégies de gestion et de développement de cette activité en plein essor.

Cet article présente ce SIG et les premiers résultats qui en sont issus. Le diagnostic du dispositif actuel d'accueil de la plaisance dans le Finistère est exposé. Les perspectives offertes par l'outil pour la gestion, l'interrogation, la mise à jour et la diffusion des données relatives à la plaisance ainsi que pour leur analyse en regard de données environnementales ou socio-économiques produites par des organismes variés sont également décrites et discutées.

\section{MoTS CLÉS : Finistère - Observatoire - Plaisance - SIG.}

\section{ABSTRACT}

\section{A GIS dedicated to boating in the Finistère (France)}

Recreational boating is regarded as an asset for economic development in the Département du Finistère, according to its direct incomes. It is seen also as a relevant medium to promote the image of the Département, especially in terms of environment quality and tourism facilities. However, until today this activity has developped without global planning, despite its spreading influence on coastal zone infrastructures and users.

While promoting the development of recreational boating in the Département, Nautisme en Finistère, aims at improving its organization and its integration in the local socio-economical and environmental context. Indeed, NEF has ordered and coordinated several 
complementary studies to increase knowledge in recreational boating and its dedicated infrastructures in the Finistère. The data collected have been integrated into a GIS that provides a consistent frame for the implementation of an observatory dedicated to boating that will be used for the definition of management and development strategies of this thriving activity.

This paper deals with presenting this GIS and its first outputs. The diagnosis of the Département capacities and facilities for boating is presented. The GIS potentialities for managing and spreading data about boating, but as well for cross-analysis with socio-economical and environmental data are also described and discussed.

KEY WORDS : Boating - Finistère - France - GIS - Observatory.

Par la qualité de ses paysages et la diversité de ses conditions nautiques, le Finistère est sans conteste un département attrayant pour la plaisance. Celle-ci constitue d'ailleurs un élément emblématique de son image et, au-delà, constitue un réel atout de développement économique. Si l'emploi direct est relativement limité dans les 12 principaux ports (68 emplois directs en équivalent temps plein, dont $46 \%$ de permanents et $54 \%$ de saisonniers), il faut rappeler que 10 places de ports génèrent un emploi dans différentes entreprises liées à la plaisance, ce qui correspond à environ 600 emplois dans le Département (CG29, NEF, 2000). La plaisance est également un outil de promotion touristique, particulièrement dans certaines petites communes littorales où elle peut constituer la principale activité touristique et donc un atout de développement local essentiel.

Pourtant, l'activité de plaisance s'est développée jusqu’à présent avec ampleur et rapidité mais sans souci de mettre en place une organisation globale prenant en compte une dimension géographique. Aujourd'hui, une structuration devient nécessaire face à l'emprise spatiale croissante de la plaisance et à la pression qui en découle : manque de places et développement de listes d'attente dans les ports, multiplication des mouillages non autorisés, difficultés de création de ports ex nihilo, conflits d'usage à terre et en mer, impacts environnementaux multiples...

Les acteurs de la plaisance mais également les gestionnaires d'aires marines protégées cherchent depuis plusieurs années à mieux comprendre l'activité touristique et à apporter des solutions aux problèmes qui se posent actuellement (Brigand, 2004). Regroupant de façon paritaire le Conseil Général du Finistère et les associations nautiques finistériennes, Nautisme En Finistère (NEF), une association de loi 1901, joue un rôle fédérateur prépondérant pour l'organisation et le développement de la plaisance dans le département ${ }^{1}$. Son action a en effet pour but de réaliser des études globales sur l'évolution de la plaisance dans le Finistère, pour coordonner le secteur et les différentes activités qui en dépendent, pour rationaliser les équipements et les infrastructures liées, pour proposer de nouveaux produits ou de nouvelles activités, et enfin pour assurer la promotion de l'ensemble. À ce titre, NEF a engagé plusieurs études complémentaires visant à améliorer la connaissance de cette activité relativement mal connue et de ses infrastructures. En vue de faciliter l'exploitation et la diffusion des données collectées, un SIG dédié à la plaisance a ainsi été développé. Il constitue la base d'un observatoire de la plaisance mis en œuvre au sein de NEF afin de pouvoir définir les stratégies de gestion et de développement de cette activité en plein essor. Cet article présente le SIG mis en œuvre dans ce contexte ainsi que les premiers résultats qui en sont issus. 


\section{Contexte de l'étude : une activité encore mal connue}

À l'exception de quelques études générales (Mauvais, 1991; Bernard, 1993) la plaisance est longtemps restée peu connue à l'échelle départementale. La forte variabilité spatio-temporelle de cette activité et le fait qu'elle ne soit pas systématiquement encadrée, rend difficile une évaluation fiable des effectifs d'embarcations, de leurs lieux de mouillages et des secteurs fréquentés. Par exemple, bien que le nombre total d'immatriculations délivrées par les Affaires maritimes s'élevait à 79268 au 31 août 2002, l'estimation réalisée à la même époque par l'Association des Ports de Plaisance de Bretagne (APPB, 2002) faisait état de seulement 12371 mouillages au sein des concessions portuaires. Cette apparente incohérence provient d'une part de la surestimation du nombre d'embarcations par les immatriculations qui concernent aussi bien les embarcations qui naviguent que celles qui sont désarmées ou détruites et, d'autre part, de leur sous-estimation par le dénombrement des concessions de mouillage qui ne tient pas compte des mouillages « forains » et des embarcations qui ne nécessitent pas d'un mouillage (bateaux sur remorque par exemple). De fait, un état des lieux exhaustif des mouillages est apparu indispensable dans la perspective de définir une politique départementale de la plaisance et une stratégie d'aménagement du littoral susceptible de pouvoir répondre à une demande croissante tout en respectant l'environnement littoral et ses usagers.

Un premier état des lieux a été réalisé par NEF (Gonthier, 2003), dans la perspective de mettre en place un Schéma Départemental de la plaisance, comme prévu dans le Livre Bleu 2000-2006 (CG29 et NEF, 2000). Réalisée en collaboration avec les principaux acteurs du nautisme dans le Finistère (gestionnaires des ports et des mouillages organisés, collectivités locales, Chambres de Commerce et d'Industrie, Sociétés d'Economie Mixte, associations d'usagers, services de l'État), cette étude a servi de support à la définition des grandes orientations stratégiques adoptées pour l'organisation et le développement de la plaisance dans le département. Le Schéma départemental de la plaisance (NEF, 2006) a ainsi permis de faire évoluer l'attribution des aides départementales, en passant d'un taux de subvention fixe, quel que soit le type d'aménagement prévu, à des aides ciblées sur des aménagements jugés prioritaires. Des investissements indispensables bien que non immédiatement rentables ont été privilégiés : équipements de mise à l'eau de bateaux « transportables », organisation ou réorganisation de mouillages, traitement des eaux de carénage, création ou amélioration de structures d'accueil, reconversion des friches portuaires, aménagements paysagers et balisage. Au cours de cet état des lieux, la flotte active a été estimée entre 43000 et 46000 embarcations.

Pour disposer d'un État des lieux des mouillages organisés et forains utilisés par les plaisanciers le long des côtes finistériennes, une seconde étude a été confiée au laboratoire Géomer (LETG UMR CNRS6554). Une méthode originale a été imaginée pour parvenir à une évaluation précise du nombre de mouillages et du type d'embarcations présentes à différentes périodes de l'année sur le littoral finistérien. Reposant sur trois survols aériens à basse altitude réalisés en juillet 2004, février et mai 2005, cette méthode a permis pour la première fois de produire une estimation fiable et instantanée du nombre de mouillage et du type d'embarcations présentes sur l'ensemble du littoral finistérien et d'en avoir une représentation spatiale précise et incontestable (Brigand et al 2005). À titre d'exemple, 21238 embarcations ont été comptabilisées le 6 juillet 2004, date de la plus forte fréquentation observée. Chaque type d'embarcation a été identifié et classé en neuf catégories : voiliers, voile légère, embarcations à moteur, canots pneumatiques, pêche professionnelle, autres, indéterminé, corps-morts inoccupés. En outre cette étude, présentée sous la forme d'un atlas communal, a permis de déterminer géographiquement tous les ports, abris et mouillages autorisés ou forains. Ces données présentées lors de deux réunions publiques ont été à la base des réflexions et des travaux qui ont été réalisés par la suite.

Dans la continuité de l'étude de Géomer, un Inventaire des infrastructures de plaisance à l'échelle du Finistère, a été réalisé, à la demande de NEF, par l'association PATEL (2006) ${ }^{2}$ constituée par 
les étudiants de l'option «Expertise et Gestion de l'Environnement Littoral (EGEL)» du Master «Sciences de la mer et du littoral » (SML) de l'Institut Universitaire Européen de la Mer (IUEM) basé à Plouzané (Finistère). Le but de cette étude est d'identifier les mouillages offrant les meilleures conditions d'accueil aux plaisanciers afin de promouvoir la plaisance d'escale et le passage. Une analyse approfondie des données collectées lors de cet inventaire a permis de mettre en évidence les atouts et les limites actuelles de chaque mouillage et de formuler des propositions, site par site - ainsi qu'à l'échelle départementale - pour améliorer les conditions d'escale des plaisanciers et l'intégration de l'activité plaisance dans son environnement naturel et anthropique.

Pour faciliter l'exploitation de ces données et en permettre une large diffusion auprès de l'ensemble des acteurs du nautisme dans le Finistère, les données produites lors de ces études ont été intégrées à un Système d'Information Géographique (SIG) qui permet d'alimenter l'observatoire de la plaisance mis en place par NEF.

\section{Un SIG dédié à la plaisance : une application originale}

Imaginés dès les années 1960, développés en parallèle avec les progrès de l'informatique dans les années 1970-1980, les systèmes d'information géographique offrent des fonctionnalités variées pour gérer, analyser et diffuser de l'information géographique (Coppock \& Rhind, 1991). Ils apparaissent d'autant plus pertinents que l'on considère généralement que plus de $80 \%$ des données peuvent être rattachées à une localisation ou à un objet géographique (Longley et al., 1999).

Les SIG permettent en effet d'intégrer des données de nature et de sources variées dans des bases d'information géographique cohérentes; de gérer ces données afin d'en faciliter l'accès aux utilisateurs souhaités; de mettre en œuvre des outils puissants pour le traitement statistique et l'analyse spatiale de ces données et, enfin, de les éditer sous différentes formes (cartes, tableaux, rapports...) afin d'en permettre une large diffusion. De par ces fonctions variées, et bénéficiant de la démocratisation des outils informatiques, l'utilisation des SIG s'est largement répandue hors du giron de la recherche, au sein des entreprises, des institutions publiques et des collectivités territoriales à partir des années 1990. Les applications qui y ont été développées couvrent de nombreux domaines opérationnels parmi lesquels on peut citer (par exemple si l'on se restreint à la France) l'aménagement du territoire et l'urbanisme (Essevaz-Roulet, 2003), la gestion des transports (Mondou, 2001), le suivi et la protection de l'environnement (Gourmelon et Robin, 2005), la gestion des risques et la sécurité (Le Berre et al., 2008), etc.

Les SIG offrent ainsi des potentialités intéressantes pour le suivi et l'étude d'une activité comme la plaisance. Cette activité présente en effet une forte variabilité spatiale et temporelle (notamment saisonnière) et nécessite un suivi quasi permanent. Les données la concernant doivent donc faire l'objet d'une mise à jour régulière afin de pouvoir disposer d'un état toujours actualisé de l'activité. Également, en plus des données la concernant directement (flotte, infrastructures, etc.), l'appréhension des enjeux liés à la plaisance nécessite de recourir à des données variées concernant son contexte socio-économique (secteur économique lié, usages « concurrents » du littoral par exemple), et son contexte environnemental qui détermine à la fois des contraintes pour le déroulement de la plaisance (conditions de navigation, nature du littoral) et qui, en retour, est susceptible de pâtir d'un développement mal contrôlé de cette activité (impact potentiel sur des habitats ou des espèces à forte valeur patrimoniale).

Pourtant, les applications SIG dédiées à la plaisance restent rares. Dans la majeure partie des cas, ils constituent un outil d'aide à la navigation en complément (mais jamais en remplacement) ${ }^{3}$ des instructions nautiques, en mettant par exemple à disposition des plaisanciers des informations concernant les ports et structures d'accueil, la nature et la qualité des services qu'ils sont suscep-

3. En raison de leur caractère officiel et réglementaire, les instructions nautiques sont strictement éditées par les services hydrographiques (en France le SHOM). Elles doivent être utilisées en complément des cartes marines officielles et des livres de feux (cf. http://www.shom.fr). 
tibles d'y trouver et, parfois, concernant les conditions de navigation ${ }^{4}$. Mais les SIG existants ne sont généralement pas spécifiquement dédiées à la plaisance qui constitue un domaine thématique parmi d'autres dans des outils destinés le plus souvent à la gestion portuaire (Le Berre et al., 2002 $)^{5}$. Par ailleurs, la plaisance fait naturellement partie des activités prises en compte dans les projets de la gestion intégrée du littoral. Mais dans ce cas, ce sont essentiellement les infrastructures et la réglementation maritime qui sont prises en compte (Brody et al., 2004; Fann, 2007 ; Préfecture du Morbihan, 2006; Villa et al., 2001). Peu de données spécifiques et précises sont mobilisées concernant par exemple la flotte et les zones fréquentées. Sans doute moins encadrée que d'autres activités telles que la pêche ou le transport maritime (Le Tixerant, 2004), la plaisance constitue en effet une activité difficile à étudier en raison de la faible disponibilité de données exhaustives et actualisées. Les solutions proposées résident alors dans des enquêtes sur le terrain (Pelot et al., 2004), ou par voie postale (Sidman et al., 2005) ou bien dans la mise en œuvre de suivis aériens comme c'est le cas sur les sites de Porquerolles et de l'archipel de Chausey dans le cadre des observatoires « Bountîles » de la fréquentation mis en place depuis plusieurs années par le laboratoire Géomer (Brigand et Le Berre, 2006). L'outil SIG est alors employé pour identifier les zones les plus fréquentées et pour inventorier les embarcations en vue d'analyser les interactions de la plaisance avec les autres usages du littoral.

Dans ce contexte, NEF a souhaité se doter d'un SIG visant à rassembler sous une forme cohérente les données relatives à la plaisance dans le département du Finistère. Cet état des lieux doit permettre de répondre à plusieurs objectifs :

- identifier les lacunes en terme de connaissance de cette activité et mettre en place les études nécessaires ou les partenariats pertinents pour les combler;

- établir un diagnostic général des infrastructures liées à la plaisance afin d'optimiser l'offre dans ce domaine et d'en déterminer les points faibles et les lacunes;

- évaluer finement l'importance et la nature de la flotte de plaisance dans le Finistère, ainsi que les usages qui lui sont liés et les enjeux socio-économiques et environnementaux qu'ils induisent.

- Accorder l'offre départementale avec cette demande, d'abord en optimisant les structures existantes puis, si nécessaire, en développant de nouvelles installations;

- Alimenter un observatoire du nautisme à partir duquel seront établis les Schémas départementaux de la plaisance destinés à encadrer le développement de cette activité, pour l'encourager son développement dans le respect de l'environnement et des autres usages du littoral.

Par conséquent, l'outil dont NEF a souhaité se doter est avant tout conçu pour l'aide à la gestion de la plaisance à destination des collectivités (le département, les communautés de communes et le pays touristiques) et des administrations (l'équipement en particulier) compétentes dans ce domaine.

\section{Méthodologie}

En tout, une vingtaine d'étudiants ont collaboré à cette étude qui, sur le terrain, a consisté en un inventaire réalisé à partir des zones de mouillages préalablement identifiées par voie aérienne dans le cadre de l'étude réalisée par Géomer. Les investigations menées ont permis de recenser les infrastructures et les services disponibles pour le plaisancier sur l'ensemble des mouillages organisés, forains ou estivaux. La grande quantité de données collectées (plus de 110 critères

4. À ce sujet voir, par exemple le site Ohio Boating Areas [http://www.dnr.state.oh.us/tabid/2297/default.aspx], le Marine Resources GIS du Florida Fish and Wildlife Research Institute [http://ocean.floridamarine.org/mrgis/viewer.htm], ou bien, en France, l'Almanach du marin breton [http://www.marinbreton.com] qui propose un Guide de navigation grand public basé sur l'orthophotographie littorale permettant d'identifier les chenaux d'accès aux ports de plaisance, les balises, la capitainerie et d'autres équipements.

5. Cf. également le diagnostic des ports de la Communauté urbaine de Marseille Provence Métropole par la Sogreah (sogreah.fr). 
relevés pour les 369 mouillages étudiés) a ensuite été archivée au sein d’une base de donnée sous Access structurée en huit thèmes : parkings, services techniques, commerces, commodités, cafésRestaurants, cales, environnement urbain. Les données ont ensuite été intégrées sous ArcView 8 au SIG créé par Géomer pour NEF, sous forme de couches thématiques décrivant les aires de mouillages, les cales et les parkings (tableau 1).

En complément, une base de données photographique a été élaborée sous AcdSee. Outre une vue générale de chaque mouillage, elle intègre un cliché montrant chaque cale (422 recensées au total), les zones de stockages des annexes, les accès aux mouillages et divers autres éléments comme les pontons d'attente, les grues et les treuils de manutention, les zones de stockage des bateaux à terre, ou le type de traitement des déchets. Les photographies sont classées par catégorie, mais aussi par commune et par zone de mouillage. Pour le moment, cette base de données photographique est indépendante du SIG, mais constitue un élément essentiel de l'observatoire de la plaisance mis en place par NEF.

L'étude s'est ensuite poursuivie dans le cadre du stage d'une durée de six mois réalisé à NEF par un étudiant du master EGEL (avril-septembre 2006) afin de vérifier et de valider les données collectées, de finaliser leur intégration au SIG, de les analyser et de les éditer (Nardin, 2006). Au cours de ce stage, le SIG et les BD associées ont été déployés sur un poste de travail de NEF et la personne chargée de leur administration a été initiée à leur exploitation.

\section{Résultats}

Les outils ainsi développés permettent à NEF de disposer et de pouvoir exploiter rapidement des données complètes, actualisées et pertinentes pour alimenter la réflexion en cours sur le développement de la plaisance dans le Finistère. Des avancées significatives ont été obtenues pour rendre possible la production des résultats suivants :

- Inventaire des mouillages dans le Finistère;

- Structuration de l'offre départementale autour des mouillages les mieux équipés;

- Identification globale des points à améliorer ou à étudier ;

- Interactions entre la plaisance et l'environnement littoral;

- Proposition de nouvelles activités ou produits.

\section{InVENTAIRE DES MOUILLAGES DANS LE FinistÈRE}

Au total en 2006, on dénombre dans le Finistère, cinq ports nationaux (686 places), 16 ports départementaux (3284 places), 68 ports communaux (10916 places) soit une offre totale de près de 15000 places réparties dans 89 ports.

Sur cet ensemble, on compte 12 ports dits structurants, c'est-à-dire des ports à pontons. Une réelle dissymétrie peut être observée dans la répartition des ports structurants entre la côte nord et la côte sud (fig. 1). Elle s'explique en partie par la morphologie de la côte et par les marnages plus importants de la côte nord qui la rendent plus difficilement accessible.

Hormis les ports, les mouillages du Finistère font l'objet d'Autorisations d'Occupation Temporaires collectives (AOT) qui impliquent une déclaration auprès de la Direction Départementale de l'Équipement, et qui sont gérés majoritairement par les communes, ou bien par des associations de plaisanciers ou des centres nautiques. Au total, en 2006, on recense 41 AOT collectives, regroupant 2963 embarcations. Avec les 14886 places recensées dans les ports, cela porte l'offre officielle de mouillages dans le département à 17849. Le nombre total de mouillages recensé le 6 juillet 2004 par Géomer étant de 21 238, on peut donc estimer à 3389 le nombre d'AOT individuelles, de mouillages forains ou estivaux, les deux derniers types étant largement majoritaires. 


\begin{tabular}{|c|c|c|c|c|c|}
\hline $\begin{array}{c}\text { Couche } \\
\text { thématique }^{\text {a }}\end{array}$ & Description & Entité & Attributs & Source & Commentaire \\
\hline Sites mouillage & Localisation des mouillages & Point & $\begin{array}{l}\text { Lieu-dit, commune, } \\
\text { statut, type de } \\
\text { gestionnaire, } \\
\text { remarques }\end{array}$ & $\begin{array}{l}\text { PATEL, } \\
\text { Géomer }\end{array}$ & \\
\hline Zones mouillage & Emprise des zones mouillages & Polygone & $\begin{array}{l}\text { Lieu-dit, commune, } \\
\text { type de gestionnaire, } \\
\text { nombre de places }\end{array}$ & Géomer & \\
\hline Parkings & $\begin{array}{l}\text { Localisation des parkings situés à } \\
\text { proximité des mouillages }\end{array}$ & Point & $\begin{array}{l}\text { Code mouillage, } \\
\text { commune, revêtement, } \\
\text { accessibilité, utilisation }\end{array}$ & PATEL & \\
\hline Cales & $\begin{array}{l}\text { Localisation des cales } \\
\text { permettant l'embarquement et le } \\
\text { débarquement de bateaux }\end{array}$ & Point & $\begin{array}{l}\text { Code mouillage, } \\
\text { commune, état, } \\
\text { largeur, accessibilité, } \\
\text { équipement }\end{array}$ & PATEL & \\
\hline $\begin{array}{l}\text { Services } \\
\text { techniques }\end{array}$ & $\begin{array}{l}\text { Services techniques liés à } \\
\text { la plaisance (grutage, point } \\
\text { essence, aire de carénage, } \\
\text { magasin d'équipement nautique, } \\
\text { dépannage, stockage de } \\
\text { bateaux...) proposés à proximité } \\
\text { de la zone de mouillage }\end{array}$ & Point & $\begin{array}{l}\text { Code mouillage, } \\
\text { commune, services } \\
\text { proposés }\end{array}$ & PATEL & \\
\hline $\begin{array}{l}\text { Restauration, } \\
\text { cafés }\end{array}$ & $\begin{array}{l}\text { Établissements de restauration et } \\
\text { débit de boisson situés à proximité } \\
\text { de la zone de mouillage }\end{array}$ & Point & $\begin{array}{l}\text { Code mouillage, } \\
\text { commune }\end{array}$ & PATEL & $\begin{array}{l}\text { Présencelabsence } \\
\text { seule indiquée }\end{array}$ \\
\hline Hébergement & $\begin{array}{l}\text { Hébergements situés à proximité } \\
\text { de la zone de mouillage }\end{array}$ & Point & $\begin{array}{l}\text { Code mouillage, } \\
\text { commune }\end{array}$ & PATEL & $\begin{array}{l}\text { Présencelabsence } \\
\text { seule indiquée }\end{array}$ \\
\hline Commodités & $\begin{array}{l}\text { Commodités (point info, } \\
\text { sanitaires, douches, déchetterie) à } \\
\text { proximité de la zone de mouillage }\end{array}$ & Point & $\begin{array}{l}\text { Code mouillage, } \\
\text { commune, type de } \\
\text { commodités }\end{array}$ & PATEL & \\
\hline Commerces & $\begin{array}{l}\text { Commerces (épicerie, point } \\
\text { argent, commerces divers) situés à } \\
\text { proximité de la zone de mouillage }\end{array}$ & Point & $\begin{array}{l}\text { Code mouillage, } \\
\text { commune }\end{array}$ & PATEL & $\begin{array}{l}\text { Présencelabsence } \\
\text { seule indiquée }\end{array}$ \\
\hline $\begin{array}{l}\text { Trait de côte } \\
1 / 25000\end{array}$ & $\begin{array}{l}\text { Trait de côte de la France } \\
\text { métropolitaine du SHOM, corrigé }\end{array}$ & Polygone & Aucun & $\begin{array}{l}\text { SHOM, } \\
\text { TCIFMS }\end{array}$ & $\begin{array}{l}\text { Trait de côte de } \\
\text { référence }\end{array}$ \\
\hline $\begin{array}{l}\text { Trait de côte } \\
1 / 100000\end{array}$ & $\begin{array}{l}\text { Trait de côte de la France } \\
\text { métropolitaine du SHOM, corrigé } \\
\text { et généralisé }\end{array}$ & Polygone & Aucun & $\begin{array}{l}\text { SHOM, } \\
\text { TCIFMS }\end{array}$ & $\begin{array}{l}\text { Trait de côte } \\
\text { simplifié pour la } \\
\text { cartographie des } \\
\text { données }\end{array}$ \\
\hline $\begin{array}{l}\text { Ortholittorale } \\
2000\end{array}$ & $\begin{array}{l}\text { Orthophotographie littorale du } \\
\text { SIG interservice (SIGI) }\end{array}$ & $\begin{array}{l}\text { Fichier } \\
\text { image }\end{array}$ & Aucun & $\begin{array}{l}\text { CETE } \\
\text { Normandie- } \\
\text { Centre }\end{array}$ & Téléchargeable ${ }^{\mathrm{b}}$ \\
\hline $\begin{array}{l}\text { Protection de } \\
\text { l'environnement }\end{array}$ & $\begin{array}{l}\text { Limites réglementaires de } \\
\text { protection de l'environnement }\end{array}$ & Polygone & $\begin{array}{l}\text { Nature de la protection } \\
\text { (Site classé / inscrit, } \\
\text { zone Natura } 2000, \\
\text { Réserve naturelle,...) }\end{array}$ & $\begin{array}{l}\text { DIREN } \\
\text { Bretagne }\end{array}$ & Téléchargeable \\
\hline Habitats marins & $\begin{array}{l}\text { Habitats marins prioritaires au } \\
\text { titre de la Directive Habitat }\end{array}$ & Polygone & $\begin{array}{l}\text { Type d'habitat (herbier } \\
\text { à zostère, maerl) }\end{array}$ & REBENT & Téléchargeable ${ }^{\mathrm{d}}$ \\
\hline
\end{tabular}

a. Seules les données exploitées dans le cadre de cette étude figurent dans ce tableau. L'intégration au SIG de NEF des données disponibles relatives à la réglementation et aux activités maritimes est programmée à court terme.

b. [www.geolittoral.equipement.gouv.fr].

c. [http://www.bretagne.ecologie.gouv.fr].

d. Voir site du Réseau Benthique [http://www.rebent.org/].

Tableau 1 : Dictionnaire des données intégrées au SIG de Nautisme en Finistère Data dictionary of the NEF GIS

Cependant des projets d'extension ou de création de ports sont en cours. On compte ainsi quatre créations de ports en projet plus ou moins aboutis : L'Aber-Wrac'h à Lannilis (250 places), Port du Château à Brest (600 places), Primel à Plougasnou (600 places) et Roscoff-Bloscon (600 places). 


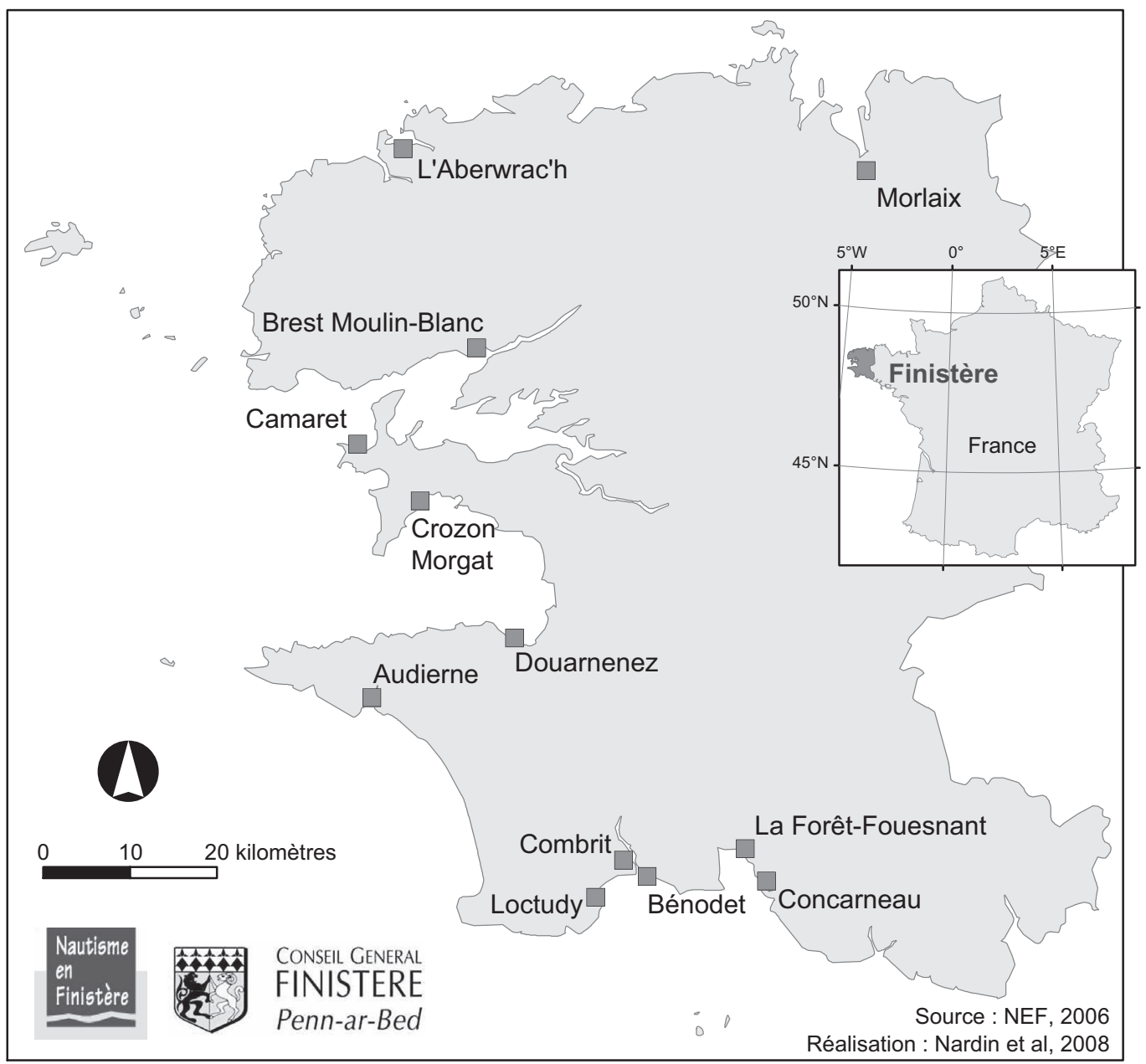

Figure 1 : Les ports structurants du Finistère Main recreational harbours of the Finistère

Considérant la difficulté de mise sur pied de nouveaux projets portuaires, la tendance est à l'agrandissement ou la reconversion de ports existants. Il existe à l'heure actuelle cinq projets de ce type : Camaret (10 places), Port-la-Forêt (30 places), Lesconil (40 places), Tréboul/Port-Rhu (150 places) et l'extension de Crozon-Morgat (environ 150 places). Un total de 2300 places devrait ainsi permettre de répondre en partie au problème des listes d'attente. Pour le reste, les créations de ports ex-nihilo tendant à devenir exceptionnelles, et les projets d'extension de plus en plus rares en raison de contraintes notamment juridiques et environnementales, de nouvelles solutions doivent être recherchées pour répondre à la demande de places. Il pourrait s'agir, par exemple, de la densification des zones de mouillage, de la reconversion de zones portuaires ou de la réalisation de ports à sec, bien que les contraintes soient également nombreuses pour ce type d'aménagement.

\section{IDENTIFICATION DES MOUILLAGES LES MIEUX ÉQUIPÉS}

Parmi les 369 zones de mouillages répertoriées dans l'étude, 88 ont été sélectionnées en fonction de leur niveau d'équipement afin de constituer un maillage régulier le long des côtes finistériennes. La 
méthode retenue pour ce faire repose sur la somme des équipements présents à proximité de la zone de mouillage, c'est-à-dire dans un rayon de 500 mètres. Les mouillages sont ainsi côtés entre 2 et 18 , ceux dont la valeur est supérieure à 8 ont été retenus (fig. 2). Cette première sélection, réalisée à partir du calcul de «zones tampon » et de requêtes sous SIG, permet de déterminer les zones de mouillage partiellement ou bien équipées pour permettre un accueil optimal des plaisanciers. La connaissance acquise par NEF sur le terrain, éventuellement complétée au cours de l'étude, a ensuite permis de réduire cette sélection en tenant compte de critères pragmatiques de navigation et de sécurité : accessibilité au mouillage, abris de la houle, sécurité d'escale, espacement entre les zones de mouillage.

Certains sites incomplètement équipés ont ainsi été retenus au vu de leur position stratégique (l'anse du Dellec à Plouzané, à l'entrée de la rade de Brest), de leur qualité de mouillage (Port du Vil dans l'Aber Benoît), ou encore de leur potentiel de développement (anse de Pouldohan à Trégunc). À l'inverse, certains mouillages possédant a priori un bon niveau d'infrastructure ont été éliminés pour des raisons de sécurité, comme le site de Mousterlin dans la commune de Fouesnant (mauvaises conditions d'accès ou d'abris), ou bien de la concurrence de mouillages proches mieux équipés, notamment dans l'Aber Wrac'h et dans l'Aber Benoît.

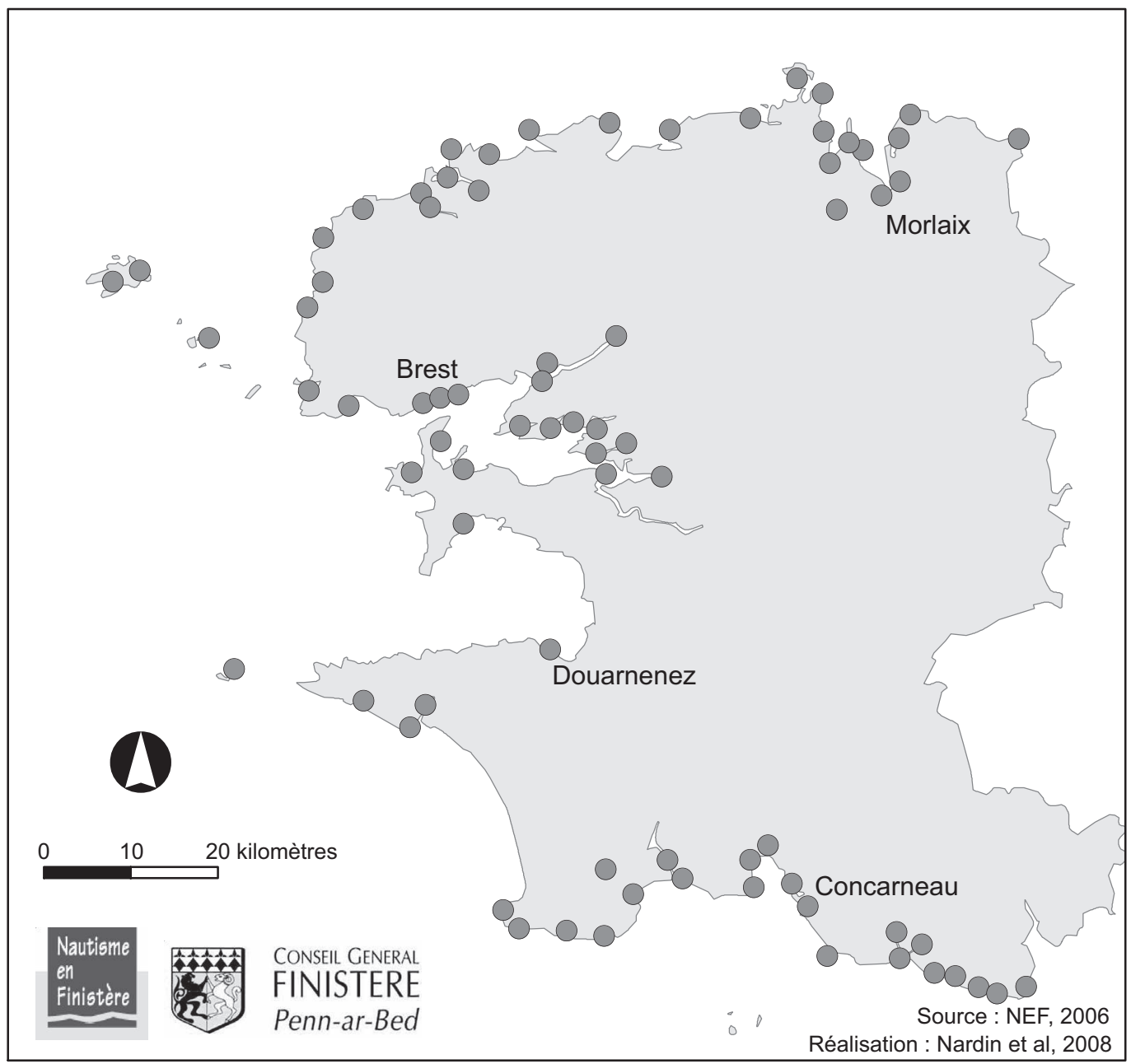

Figure 2 : Principaux mouillages identifiés d'après leur niveau d'équipement Main mooring areas as identified according to the quality of their facilities 
L'exploitation des données par cette méthode permet ainsi d'identifier un réseau de mouillages sûrs et bien équipés. Certains secteurs en restent cependant dépourvus du fait de la configuration de leurs côtes et de leur exposition aux vents dominants (baie d'Audierne, Cap Sizun, fond de la baie de Douarnenez entre Morgat et Douarnenez).

Pour présenter ce réseau de mouillages «structurants », un atlas a été réalisé. Il rassemble les fiches descriptives de chaque mouillage organisées en deux parties (fig. 3) :

- Une analyse synthétique du mouillage, divisée en trois rubriques : atouts et statut du mouillage (capacité d'accueil officielle, conditions de mouillage et d'accès, possibilités de stockage des bateaux et des annexes, présence d'un office du tourisme ou d'un centre nautique); limites et précautions particulières (inventaires écologiques, réglementations environnementales, accès, mise à l'eau et conditions de mouillage, infrastructures, situation); propositions d'aménagement du plan d'eau et des infrastructures ou d'amélioration des services à destination des plaisanciers.

- Une carte sur laquelle sont représentés l'extension du mouillage et le nombre de bateaux présents (tous types d'embarcation confondus) lors du survol aériens du 6 juillet 2004 (Géomer, 2005), les cales de mise à l'eau classées selon un code couleur, les parkings et, sous forme de pictogrammes, les services et les infrastructures disponibles dans un rayon de $500 \mathrm{~m}$ autour du mouillage (sanitaires, collecte des déchets, restauration... ${ }^{6}$.

Ces fiches permettent ainsi de disposer sous une forme synthétique d’informations relatives à la densité de bateaux et à l'emprise spatiale du mouillage, ainsi qu'à l'état des infrastructures et des services disponibles sur les principaux mouillages du Finistère. Elles permettent, par exemple, de comparer rapidement les données officielles avec le nombre de bateaux et de mouillages recensés sur un site. Pour le gestionnaire, elles permettent de connaître le niveau d'accueil de chaque mouillage et ainsi d'en envisager l'amélioration, mais surtout de pouvoir disposer de données pertinentes pour une réflexion à l'échelle du département. Potentiellement, elles pourraient également apporter des renseignements utiles aux plaisanciers, en complément des instructions nautiques, sur les services disponibles dans leurs lieux d'escale potentiels. Cependant, cette option n'est pas envisagée par NEF pour le moment.

L'inventaire réalisé et mis en forme sous SIG permet également d'étudier des «produits plaisance » reposant sur une offre locative et un encadrement de l'activité par des ports et des mouillages bien équipés. Par exemple, des circuits pour des plaisanciers de différents niveaux sont à l'étude : tour du Finistère en une dizaine d'étapes (durée approximative de deux semaines), randonnées nautiques d'une semaine autour de quatre aires géographiques (baie de Morlaix, BasLéon-Ouessant, Presqu'île de Crozon-Sein-Baie de Douarnenez, Finistère-sud-Glénan), ou bien week-ends nautiques sur différents sites (baie de Morlaix, Abers, rade de Brest, sud-Finistère) ${ }^{7}$.

\section{Application opérationnelle}

Le diagnostic global réalisé à partir de l'exploitation des données intégrées au SIG permet de mettre en évidence un certain nombre de limites au dispositif actuel d'accueil de la plaisance dans le Finistère. En s'appuyant sur les constats réalisés, des propositions concrètes ont été réalisées par NEF dans deux domaines.

\section{L'ORGANISATION DES MOUILLAGES}

L’organisation peu optimale des mouillages est un constat général : 3389 mouillages ont été identifiés comme AOT individuelle, mouillage forain ou estival, et donc non organisés (d'autant que la part des AOT individuelles est minime). L'organisation des mouillages est souvent anar-

\footnotetext{
6. Cette distance correspond à environ un quart d'heure à vingt minutes de marche pour que la plaisancier (notamment en escale) puisse accéder à ces services.

7. Cf. rubrique «Les activités » sur le site du Comité Départemental du Tourisme [http://www.finistere-nautisme.com/].
} 


\section{Carantec Nord}

La Grève Blanche

Coordonnées du mouillage : N4840'29.6" - W00355.13'.9"

Atouts
- Mouillage en partie en eau
- Intérêt environnemental/patrimonial (château du Taureau, îles notamment Callot et
Louët)
- Proximité du centre ville et des commerces
Précautions/Limites

- Absence d'AOT

Mouillage en partie sur la passe aux moutons (entre l'île Callot et la pointe de

Carantec), moins abrité

- ZNIEFF 2, ZICO, Natura 2000 ( Baie de Morlaix), site classé (lle Callot)
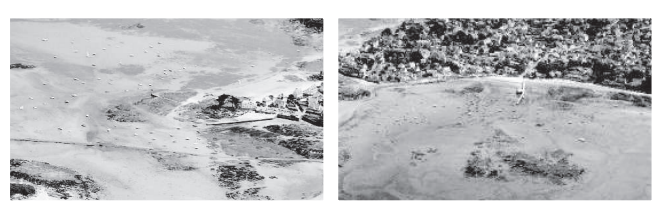

\section{Le Port}

Coordonnées du mouillage: $\mathrm{N}^{\circ} 8^{\circ} 40^{\prime} 22.4^{\prime \prime}-$ W003 $55.27^{\prime} .7^{\prime \prime}$

Atouts
- Port communal (68 places)
- Proximité du centre ville et des commerces
- Bonnes conditions d'embarquement/débarquement (quai), à partir de mi-marée
- Intérêt environnemental/patrimonial
- Possibilité de stockage des bateaux à terre
- Office du tourisme

\section{Précautions/Limites}

Limitations environnementales : ZNIEFF 2, ZICO, Natura 2000 (Baie de Morlaix) - Peu de stockage pour les annexes (12 places recensées)

- Mouillage à l'échouage, accessible environ $1 \mathrm{~h} 30$ avant et après la pleine mer

\section{Propositions d'amélioration}

\section{Propositions d'amélioration \\ - Création d'une AOT collective \\ - Regroupement des mouillages dans la partie la plus proche de la plage (éviter la passe aux moutons) \\ - Créer une zone de stockage pour les annexes}

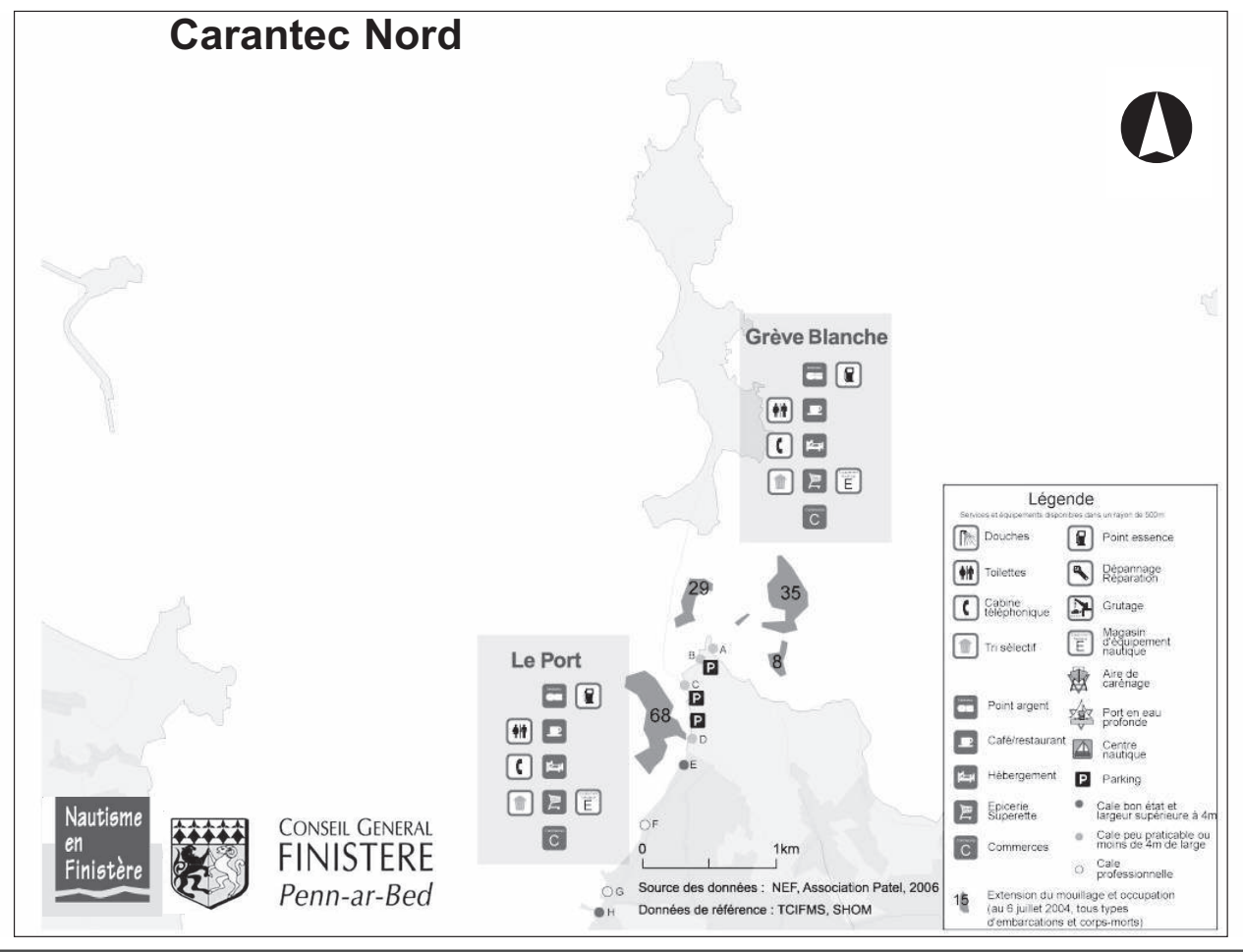

Figure 3 : Exemple de fiche descriptive de mouillage Example of a standard description of mooring area 
chique, basée sur du mouillage à évitage ${ }^{8}$, grand consommateur d'espace surtout dans les zones à fort marnage (nord Finistère), le bateau tournant librement autour de son corps-mort. De plus, certains de ces mouillages sont situés dans des sites écologiquement sensibles.

La figure 4 montre, par exemple, les risques de conflits entre les zones de mouillages et les zones Natura 2000 produites par la DIREN ${ }^{9}$ ainsi qu'avec les herbiers de zostère, prioritaires au titre de la Directive Habitat, dont la localisation peut être obtenue via le REBENT ${ }^{10}$. Dans les faits, les principales interactions possibles sont la dégradation paysagère incompatible avec certains sites classés (Térénez en Baie de Morlaix), la détérioration d'habitats prioritaires tels que des prés salés ou des herbiers de zostère (baie de Morlaix, Aber Benoît, Illien, anse de Porsmoguer, rade de Brest, Glénan), ou le dérangement de la faune (oiseaux marins notamment durant les périodes de reproduction) suite à des débarquements intempestifs sur des sites protégés (souvent insulaires). On connaît des exemples de désertion (île Dumet en Loire-Atlantique, île Méaban dans le Morbihan) liée au développement du tourisme nautique et aux débarquements fréquents et incontrôlés. Ainsi, plus d'une centaine de bateaux ont été comptabilisés certaines journées d'été à Dumet (Brigand, 1995). Il est essentiel de sensibiliser les plaisanciers, et notamment les utilisateurs de bateaux à moteur, afin qu'ils ne débarquent pas sur les îles et les sites d’hivernage (Baie de Morlaix, rivière de Pont-l'Abbé, Odet).

Suivant le même principe, l'analyse des interactions potentielles avec les autres usages du littoral peut également s'envisager à partir du SIG, à condition d'acquérir les données correspondantes auprès notamment des services de l'État, ou d'organisations professionnelles (telle que le Conseil National de la Conchyliculture par exemple).

Sur la base de ces analyses, une réorganisation des mouillages en situation potentiellement conflictuelle pourra s'envisager au coup par coup. Des solutions comme l'embossage, la densification et la réorganisation des mouillages sont à développer afin d'augmenter le nombre de places disponibles dans les sites qui le permettent, mais également de réduire l'impact environnemental et paysager dans les sites les plus sensibles. De plus, à proximité des mouillages, lorsqu'aucune infrastructure n'est disponible, les annexes sont souvent entreposées de manière anarchique. C'est le cas dans 284 des 369 sites de mouillages répertoriés. Elles ont alors une forte emprise spatiale, bloquant notamment les cales, les bords de quai ou encore les hauts de plage. L'extension ou la création de zones de stockage pour les annexes, permettrait de rationaliser l'utilisation du haut d'estran et de libérer les accès à la mer, offrant ainsi un double gain en terme de sécurité des usagers et des annexes (emprunts, départ à la mer lors des grandes marées).

Enfin, les embarcations transportables (sur remorque) posent des questions de gestion très particulières et avec une acuité accrue. Les principaux problèmes identifiés résident d'une part dans la fréquentation des cales, surtout en saison estivale, qui est susceptible de dégénérer en conflit d'usage avec les autres types de plaisanciers ou avec les pêcheurs professionnels et d'autre part dans le stockage des remorques qui encombrent les parkings des ports. De plus, en raison de la diversité des sites de mise à l'eau et, souvent, de la rapidité de ce type d'embarcation, elles permettent d'accéder à un grand nombre de sites peu accessibles avec d'autres types de bateaux, où elles sont susceptibles d'occasionner des dérangements pour certaines espèces (mammifères marins, oiseaux) jusque-là préservées. La nuisance sonore occasionnée par des moteurs souvent puissants constitue à cet égard un facteur aggravant. Parmi les cales inventoriées dans le Finistère, des requêtes effectuées à partir du SIG permettent de sélectionner celles qui présentent les spécifications nécessaires pour permettre la mise à l'eau des embarcations sur remorque (largeur, état, présence ou non d'un parking à proximité). Une première estimation des zones potentiellement

8. Les termes techniques du nautisme sont définis dans un glossaire situé à la fin de l'article.

9. Cf. site [http://www.bretagne.ecologie.gouv.fr/].

10. Cf. site du Réseau Benthique, [http://www.rebent.org/], dont les objectifs sont de « recueillir et mettre en forme les données relatives à la distribution des habitats côtiers et au suivi de leur biodiversité faunistique et floristique, pour mettre à disposition des scientifiques, des gestionnaires et du public un état des lieux pertinent et cohérent et d'en détecter les évolutions spatio-temporelles ». 


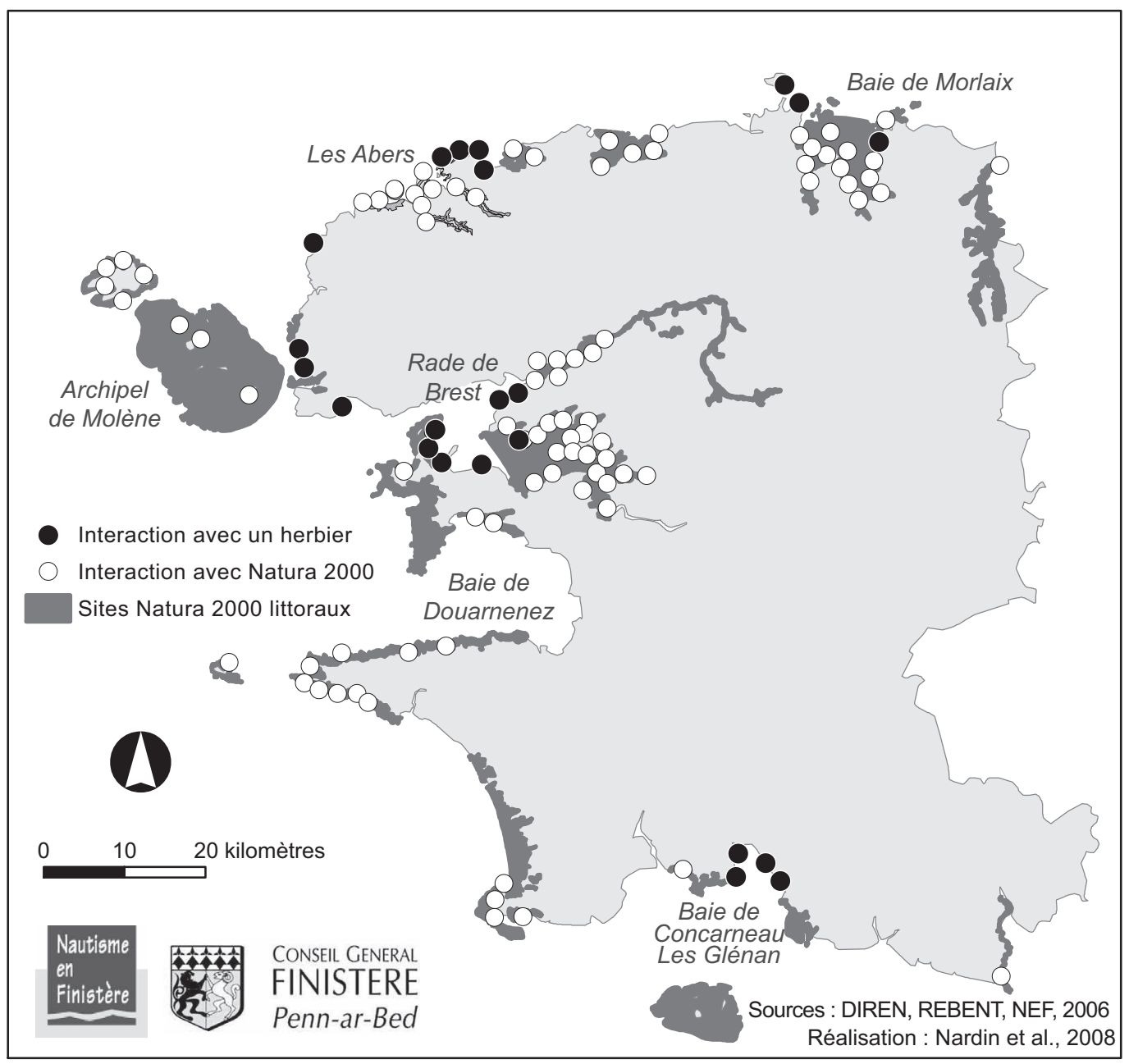

Figure 4 : Interactions potentielles entre zones de mouillages et patrimoine environnemental

Les mouillages sélectionnés sont situés dans ou à proximité (moins de $100 \mathrm{~m}$ ) des zones occupées par des herbiers ou par Natura 2000. Dans les zones de forte densité seules les principales zones de mouillages ont été représentées dans un souci de lisibilité cartographique.

Spatial interactions between mooring areas and environmental heritage

fréquentées est ainsi rendue possible afin d'identifier les secteurs d'enjeux majeurs. Cependant, il reste difficile d'évaluer le nombre de ces embarcations et leur territoire de fréquentation, a fortiori en raison de la diversité des pratiques (promenade, pratique sportive, pêche, plongée...), qui nécessitent des études spécifiques, dont plusieurs ont été engagées par NEF en 2007.

\section{LA QUALITÉ SANITAIRE DES SITES}

En plus de l'organisation de mouillages et des embarcations, les problèmes observés concernent les aspects sanitaires de gestion des déchets et des effluents toxiques liés à l'entretien des bateaux et des sanitaires. 
Dans certaines zones portuaires et dans de nombreux sites d'escales, le traitement des déchets est très loin d'être optimal : 228 sites possèdent des containers à ordures, 142 proposent le tri sélectif et seulement 32 mettent en œuvre un traitement des déchets spécifiques à la plaisance. Des solutions simples et peu coûteuses pourraient pourtant être mises en place pour améliorer la collecte et le tri des déchets (containers pour la récupération des ordures ménagères, généralisation du tri sélectif, stations de récupération des huiles usagées).

L'entretien des bateaux est également générateur de pollution par des produits souvent très toxiques, notamment les peintures anti-fooling qui contiennent des biocides à base de Tributylétain (TBT, interdit depuis le 17 janvier 1981), de plomb, de cuivre... À l'heure actuelle, peu de cales et d'aires de carénage conformes aux normes environnementales sont disponibles dans le Département. En effet, seulement 11 sites sont équipés de ce type d'infrastructure (fig. 5). Au contraire la pratique courante (et «traditionnelle») consiste à effectuer le carénage sur le haut estran... Or, il n'existe pas de législation spécifique au carénage, bien que les installations et les activités soient soumises à certaines réglementations définies dans le Code des Ports maritimes, le Code de l'Environnement ainsi que dans la Loi sur l'Eau du 3 janvier 1992. Dans le code de l'Environnement, l'article L.216-6 reprend une disposition de la Loi sur l'Eau en précisant que déverser ou laisser s'écouler, directement ou indirectement, des substances potentiellement nuisibles pour la santé ou pour la faune et la flore, peut donner lieu à des poursuites pouvant occasionner de fortes amendes voire un emprisonnement ${ }^{11}$. Compte tenu des impacts environnementaux générés par le carénage, auxquels s'ajoutent des conséquences juridiques, techniques et financières, il devient urgent d'augmenter l'offre en aires techniques et en cales de carénage. Considérant le prix de ces équipements, une mutualisation doit être recherchée au sein de bassins de plaisance. Mais des solutions alternatives peuvent s'envisager comme des carénages plus réguliers qui permettraient d'éviter l'application de peintures anti-fooling... en attendant l'amélioration de ces produits et des techniques mises en œuvre.

Enfin, une minorité de zones de mouillages sont équipées de sanitaires : 35 sont équipés de douches, 139 de toilettes sur les 369 sites inventoriés. Hormis le confort des plaisanciers, l'impact environnemental, notamment bactériologique peut être important. Diverses solutions adaptées aux différents types de mouillages devront être envisagées (conteneurs amovibles avec blocs sanitaires, sanitaires sur pontons) pour contribuer à améliorer à la fois la qualité propre des sites, et les perspectives du développement de l'escale et du passage.

Ces exemples montrent que l'exploitation du SIG permet de rechercher, d'identifier et de localiser les infrastructures de la plaisance existantes, tout en les replaçant dans leur contexte réglementaire et environnemental, afin de disposer des éléments de diagnostic pertinents pour améliorer, de manière coordonnée, l'offre du nautisme à l'échelle du département ainsi qu'à l'échelle des collectivités locales.

\section{DISCUSSION, PERSPECTIVES}

Acteur essentiel de la plaisance dans le Finistère, NEF s'est doté d'un système d'information géographique pour gérer ses données, pour les partager avec ses partenaires et pour les diffuser sous des formes variées aux acteurs et aux usagers de la plaisance.

L'utilisation d'un SIG pour intégrer sous une forme cohérente des données collectées sur le terrain ou obtenues auprès de différents partenaires, permet de faciliter l'analyse statistique et spatiale des enjeux de la plaisance, notamment en regard de données environnementales ou socioéconomiques produites par des organismes variés. L'archivage de la donnée sous forme numérique permet d'en faciliter la gestion, l'interrogation, la mise à jour et la diffusion. L'apport est considérable pour le suivi d'une activité telle que la plaisance qui évolue rapidement et dont les implications économiques, spatiales et environnementales sont très importantes. 


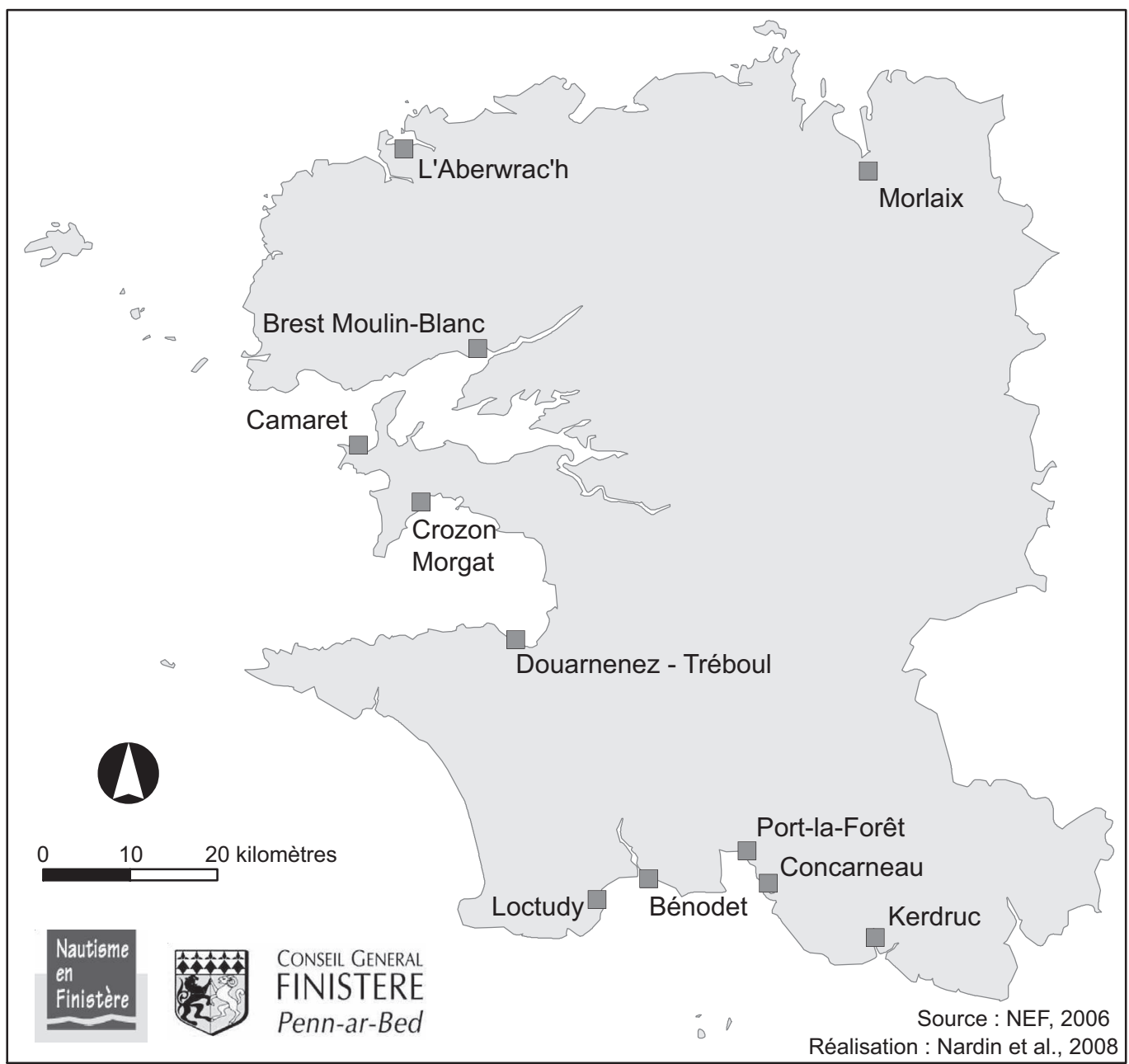

Figure 5 : Localisation des aires et des cales de carénages avec récupération des effluents dans les ports du Finistère Careening facilities in the Finistère (with sewage treatment device)

Naturellement, la mise en œuvre d'un SIG au sein d'une structure quelle qu'elle soit nécessite une adaptation structurelle et organisationnelle, notamment pour pouvoir en garantir la pérennité. Elle passe bien entendu par l'acquisition des outils (matériel et logiciels) adaptés pour répondre aux besoins de la structure. Dans le cas de NEF, un poste de travail exclusivement dédié au SIG a été acquis. Il est équipé d’ArcView $9^{\circledR}$, logiciel assez lourd à mettre en ouvre mais qui constitue une garantie de la compatibilité des données produites par NEF avec celle de ses partenaires notamment institutionnels ce qui est un élément essentiel dans ce type de projet. Il s'agit également d'un outil assez complet du point des fonctions du catalogage et de l'analyse des données, et suffisamment évolutif pour permettre d'accompagner les futurs développements envisagés par NEF. Il est a noter qu'un membre de NEF a été formé spécifiquement à l'utilisation de cet outil afin d'optimiser les traitements et de pouvoir répondre de manière optimale aux besoins de la structure.

Une autre condition nécessaire réside dans la capacité de la structure à collecter, à gérer et à mettre à jour les données nécessaires à ses besoins opérationnels et prospectifs. Elle repose à la 
fois sur son aptitude à produire et à actualiser ses propres jeux de données, mais également sur l'efficacité des partenariats qu'elle est en mesure d'établir avec les acteurs compétents et producteurs des données complémentaires pertinentes dans le domaine considéré.

Par son rôle fédérateur au sein des acteurs de la plaisance, NEF met en place les conditions partenariales et conventionnelles indispensables pour l'échange des données et pour le déblocage des moyens nécessaires à leur mise à jour. Le suivi de l'évolution de la plaisance se fera ainsi en étroite collaboration avec les acteurs de terrain et les collectivités locales, notamment les communautés de communes et les pays touristiques, de même qu'avec les administrations compétentes, notamment les services de l'Équipement. Le partenariat repose sur de l'échange de données, de l'information réciproque et sur l'expertise de NEF pour toutes les questions relatives au nautisme, en particulier dans le cadre de la mise en ouvre des SCOT des Pays côtiers du Finistère.

Par ailleurs, le suivi de l'activité de plaisance sera effectué dans le cadre d'un observatoire du nautisme appuyé par le Conseil Général du Finistère qui considère la plaisance comme un outil essentiel de développement de son potentiel touristique et économique. Conformément aux recommandations formulées dans cette étude, ce dispositif permettra de programmer des survols aériens tous les cinq ans environ pour étudier l'évolution du nombre d'embarcations présentes sur les côtes du Finistère. Ce pas de temps semble approprié d'un point de vue logistique (l'étude en elle-même constitue un travail relativement lourd) et méthodologique compte tenu de l'évolution de la plaisance (Brigand et Peuziat, 2005). Cependant, la connaissance des activités de plaisance dans le Finistère doit être complétée par des études spécifiques concernant notamment la quantité et l'utilisation des bateaux sur remorque, dont la croissance quasiment exponentielle, soulève des enjeux potentiels importants à la fois en terme d'aménagement, de gestion de conflits d'usage (notamment sur les cales), de sécurité et de respect de l'environnement (dérangement). D'autres études, à base d'enquêtes et d'entretiens auprès des usagers, seraient également nécessaires pour mieux connaître les usages des plaisanciers ainsi que leur point de vue sur les questions nautiques. L'important travail de concertation engagé par NEF notamment auprès des associations de plaisanciers va dans ce sens, mais il est évident qu'il est difficile aujourd'hui d'envisager un observatoire sans tenir compte des usagers et leurs avis (Brigand et Le Berre, 2007). Cela nécessite une approche complémentaire aux travaux actuellement en cours.

Le SIG de NEF est ainsi amené à s'étoffer par de nouvelles données et ses potentialités en terme d'analyse devraient croître en parallèle. Il constitue actuellement la base d'un observatoire du nautisme sur lequel NEF et ses partenaires institutionnels, en particulier le Conseil Général du Finistère, vont s'appuyer pour mettre en place le nouveau Schéma Départemental pour l’implantation des équipements liés à la plaisance. De plus, il sert de support à l'élaboration de nouveaux « produits » de promotion de la plaisance dans le Finistère et à l'édition de plaquettes d'information, en particulier grâce à ses fonctions de cartographie. Potentiellement, il devrait constituer un modèle pour le développement d'un outil similaire à l'échelle de la Bretagne en appui des politiques régionales de développement du tourisme et de gestion intégrée de la zone côtière.

\section{GLOSSAIRE}

Annexe : embarcation légère permettant de faire la liaison entre un bateau et le rivage. Celles-ci peuvent être stockées dans des emplacements dédiés à cet effet mais sont, le plus souvent, entreposées anarchiquement à terre ou sur l'eau

Antifooling (ou peinture antifouling) : c'est une peinture dite «antisalissures » destinée à empêcher les organismes marins de se fixer sur la coque des navires.

Carénage : le carénage est l'action de nettoyer la carène ou la coque d'un navire. Depuis la Loi sur l'eau de 1992, il est interdit d'effectuer cette action en laissant les effluents s'écouler dans le milieu naturel. Les travaux d'entretien et de réparation de la coque doivent donc s'effectuer sur des aires de carénage ou des 
cales de carénage, où les effluents sont récupérés et stockés dans des cuves avant un rejet des eaux dans le milieu. Les infrastructures les plus répandues sont les aires de carénage (photo 1). Sur les littoraux à marées, on trouve également des cales de carénage (photo 2), qui laissent quelques heures pour effectuer les réparations sur son bateau.

Embosser (un navire) : mouiller ou amarrer le bâtiment de l'avant et de l'arrière, pour le tenir dans une direction déterminée malgré le vent ou le courant (photo 3).

Mouillage à évitage : c'est le type de mouillage le plus répandu sur les côtes bretonnes. Le bateau est amarré à un corps-mort par son avant, et il évite autour de la bouée selon le vent et les courants (photo 4).

Mouillage forain : synonyme de mouillage sauvage, il n'a aucune existence légale (pas d'Autorisation d'Occupation Temporaire, AOT). Peu organisé, il est le plus souvent très consommateur d'espace.

Photo 1 : Aire de carénage de Morlaix (source : G. Nardin, 2006)

Careening device in Morlaix (source : G. Nardin, 2006)
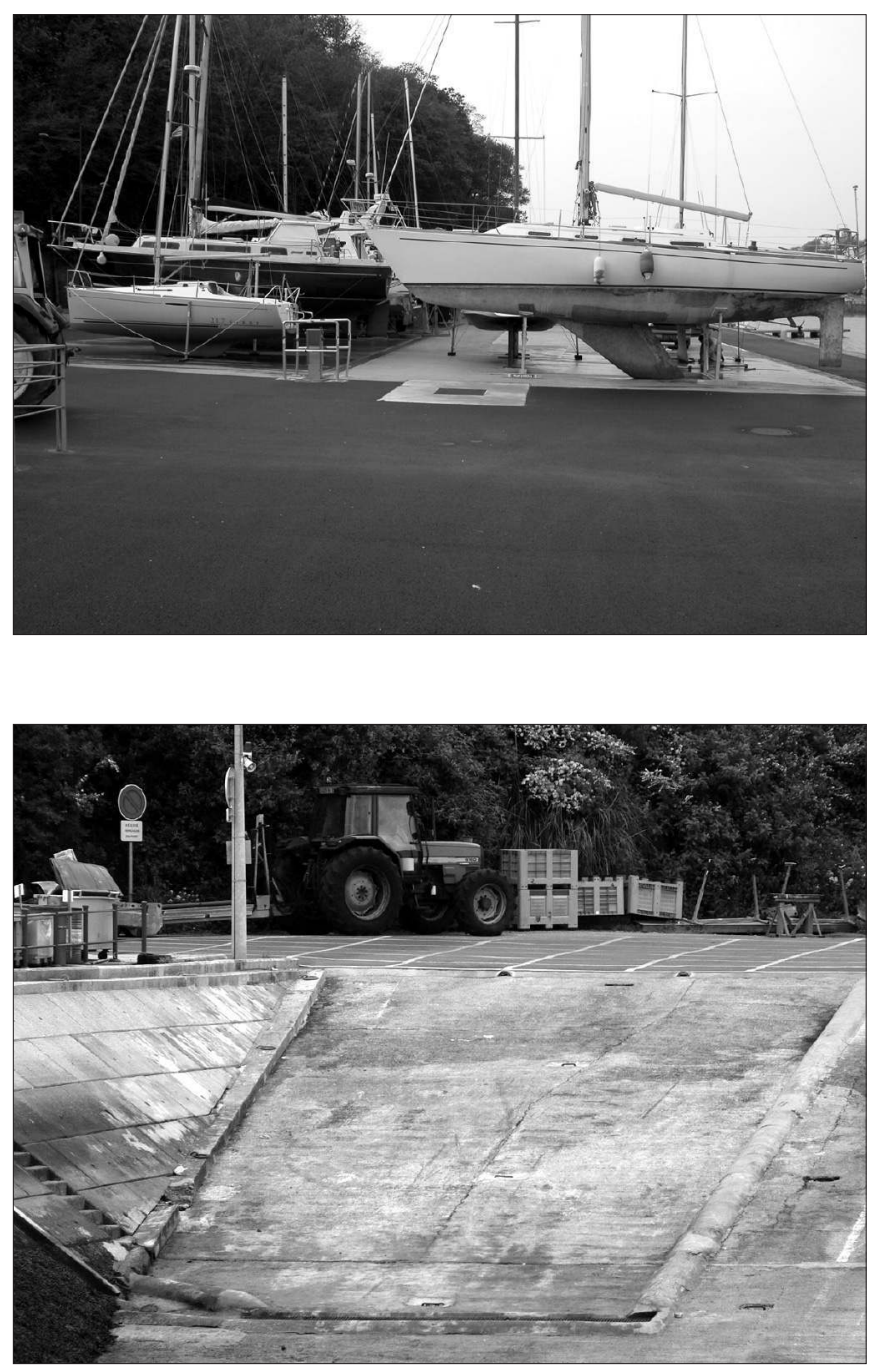

Photo 2 : Cale de carénage de Douarnenez (source : G. Nardin, 2006)

Careening ramp in

Douarnenez (source : G. Nardin, 2006) 

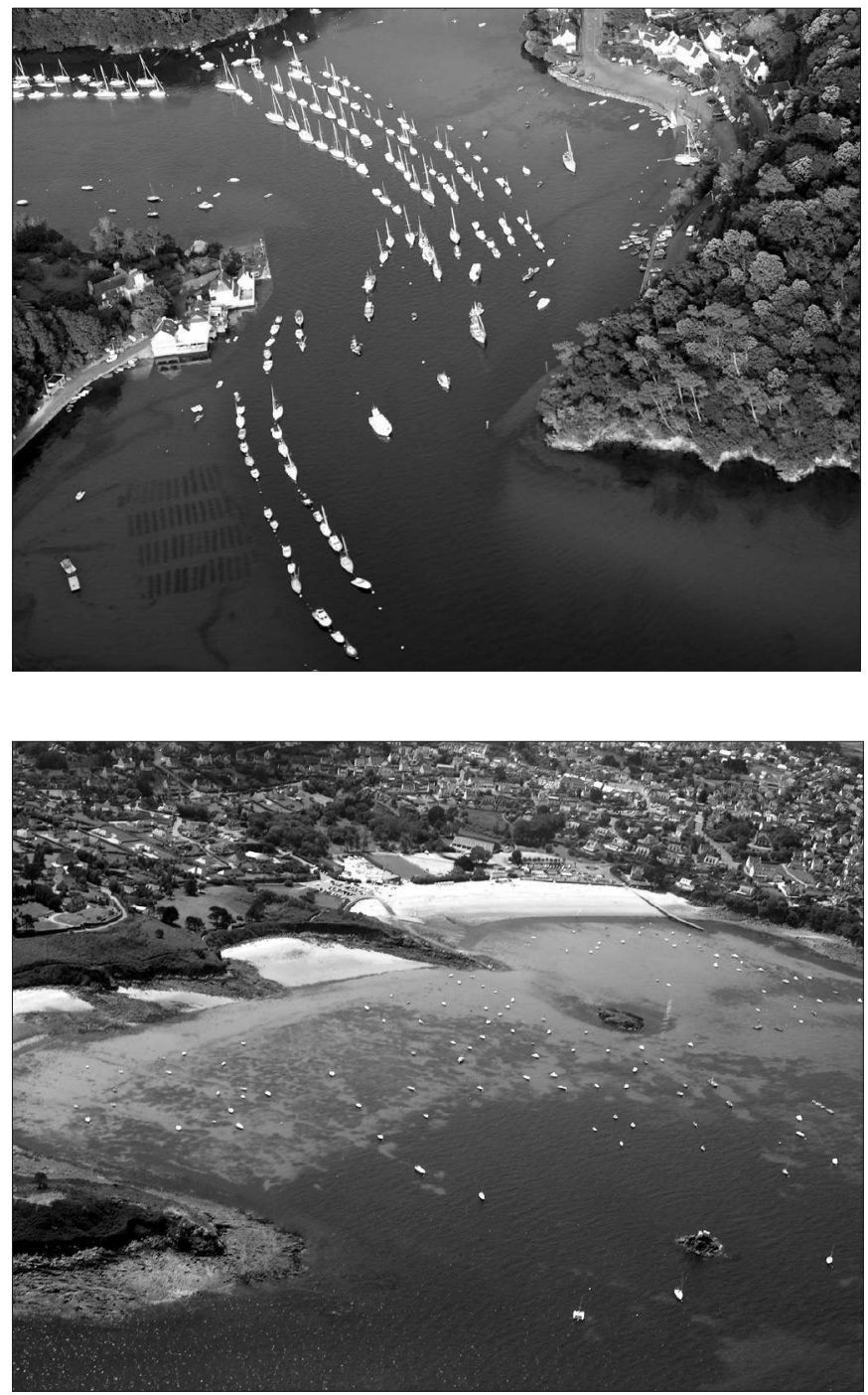

Photo 3 : Mouillage à embossage dans le Belon (source: Geomer/NEF, 2005)

Stern anchor mooring in the Belon estuary (source: Geomer/ NEF, 2005)

Photo 4 : Mouillage à évitage non organisé à Carantec (source : Geomer/NEF, 2005)

Non organized anchorage mooring at Carantec (source : Geomer/NEF, 2005) 


\section{Bibliographie}

APPB, 2002. - La plaisance en Bretagne, ports et mouillages. Rapport Mission régional ports de plaisance, Association des ports de plaisance de Bretagne, $67 \mathrm{p}$.

Association PATEL, 2006. - Inventaire des infrastructures de plaisance à l'échelle du Finistère, Rapport PATELIUEM-UBO/NEF, $237 \mathrm{p}$.

Bernard (N.), 1993. - Ports de plaisance et structuration de l'espace littoral finistérien, Thèse de doctorat de Géographie, Université de Bretagne Occidentale, Brest, 275 p.

Brigand (L.), 1995. - « Les motifs de fréquentation touristique de l'île Dumet et de l'île du Pilier », Les Cahiers du Conservatoire du littoral, $\mathrm{n}^{\circ}$ 4, p. 63-74.

Brigand (L.), 2004. - «Connaître la fréquentation nautique », Espaces naturels, n 9, p. 16-17.

Brigand (L.), Le Berre (S.) et al., 2005. - État des lieux des mouillages organisés et forains utilisés par les plaisanciers le long des côtes finistériennes, Rapport Laboratoire Géomer, Université de Bretagne Occidentale/Nautisme en Finistère, 199 p.

Brigand, (L.), Le Berre, (S.), 2006. - Etude de fréquentation de l'archipel des îles Chausey, Rapport Laboratoire Géomer, Université de Bretagne Occidentale/Conservatoire du littoral, 115 p.

—, 2007. - « Joint construction and appropriation of indicators by the users, the managers and the scientists : the example of the touristic frequentation observatory of Port-Cros and Porquerolles ", International Journal of Sustainable Development, vol. 10, n 1/2, p. 139-160.

Brigand (L.), Peuziat (I.), 2005. - « Nautisme et fréquentation des espaces sensibles. Le cas des îles et des archipels de la France métropolitaine », dans Bernard (N.) (dir.), Le nautisme, acteurs, pratiques et territoires, p. 301-319.

Brody (S. D.), Highfield (W.), Arlikatti (S.), Bierling (D. H.), Ismailova (R. M.), Lee (L.), Butzler (R.), 2004. - « Conflict on the Coast: Using Geographic Information Systems to Map Potential Environmental Disputes in Matagorda Bay, Texas », Environmental Management, vol. 34, n 1, p. 11-25.

CG29, NEF, 2000. - La filière nautique en Finistère, Livre bleu 2000-2006. Conseil général du Finistère, Nautisme en Finistère, 300 p. (synthèse de 20 p. disponible sur [www.cg29.fr/filemanager/download/103/ livre_bleu.pdf]).

Coppock (J. T.), Rhind (D. W.), 1991. - « The history of GIS », dans Maguire (D. J.), Goodchild (M. J.), RHind (D. W.) (dir.), Geographical Information Systems, principles and applications, London, Longman Scientific and Technical, vol. 1, p. 21-43.

Essevaz-Roulet (M.), 2003. - La mise en œeuvre d’un SIG dans les collectivités territoriales, Techni-cités, 257 p.

FAnN (D.), 2007. - «A geodatabase of boating ordinances », Coastal GeoTools '07, NOAA Coastal Service Center, Myrtle Beach, USA, 5-8 Mar 2007, p. 36-37.

Gonthier (Y.), 2003. - État des lieux de la plaisance en Finistère, Rapport Nautisme en Finistère, 125 p. Gourmelon (F.), Robin (M.) (dir.), 2005. - SIG et environnement côtier, Traités IGAT, éd. Hermès, 328 p.

Kerhoas (J.), 1994. - « Nautisme En Finistère », Cahiers espaces, nº 35, p 98-104.

Le Berre (I.), Metzler (N.), Gourmelon (F.), 2002. - SIG et services maritimes, étude méthodologique pour la création de couches d'information sur le littoral, CETMEF/Ministère de l'Équipement, des Transports et du Logement, 88 p.

Le Berre (I.), Quemmerais (F.), Fichaut (B.), 2008. - « Révision de l'atlas Polmar-Terre du Département de la Manche : vers un SIG opérationnel interservices ", Cybergeo, accepté décembre 2007, à paraître.

Le Tixerant (M.), 2004. - Dynamique des activités humaines en mer côtière. Application à la Mer d'Iroise, Thèse de Géographie UBO, Brest, 210 p.

Longley (P. A.), Goodchild (M. F.), Maguire (D. J.), Rhind (D. W.) (dir.), 1999. - Geographical Information System : principles and applications. London, Wiley, 1296 p.

MauvaIs (J.-L.), 1991. - Les ports de plaisance : impact sur le littoral. Ed. IFREMER, Brest, 165 p.

Mondou (V.), 2001. - «Daily mobility and adequacy of the urban transportation network a GIS application », Cybergeo, $\mathrm{n}^{\circ}$ 192, [http://www.cybergeo.en/index990.html].

Nardin (G.), 2006. - Modalités de gestion des mouillages dans le département du Finistère, mémoire Master 2 Expertise et gestion de l'environnement littoral (EGEL), IUEM-UBO, Plouzané, 175 p. 
NEF, 2006. - Schéma départemental de développement de la plaisance, Nautisme en Finistère, 75 p.

Pelot (R.), Delbridge (C.), Jakobsen (M.), Mcisaac (M.), 2004. - Maritime Activity and Risk Investigation Network. Marin report 2006, Dailhousie University, Department of Industrial Engineering, 315 p. [http:// www.marin-research.ca/pdf/MARIN_Report_2006-02_Recreational_Boating_Risk_Project.pdf].

Préfecture du Morbihan, (2006). - Documents de référence du SMVM du Golfe du Morbihan. [http://www. morbihan.pref.gouv.fr/prefecture/Actualites/dossiers_actualites/].

Sidman (C.), Swett (R.), Fik (T.), Fann (S.), Fann (D.), Sargent (B.), 2005. - A Recreational Boating Characterization for the Greater Charlotte Harbor. Florida Sea Grant, University of Florida, 94 p. [http://nsgl. gso.uri.edu/flsgp/flsgps05004.pdf].

Villa (F.), Tunesi (L.), AgARdy (T.), 2001. - « Zoning marine protected areas through spatial multiple-criteria analysis : the case of the Asinara island national marine reserve of Italy », Conservation Biology, vol. 16, n², p. 515-526.

\section{Remerciements}

Les auteurs tiennent à associer les étudiants du M2 EGEL, promotion 2005-2006, du master Sciences de la mer et du littoral de l'IUEM-UBO ayant participé à l'étude sur laquelle s'appuie cet article. Nous associons également à ce travail, en les remerciant particulièrement, MM. Jean Kerhoas et Yann Gonthier, de Nautisme en Finistère pour avoir initié et soutenu cette étude et pour avoir accueilli Guillaume Nardin lors de son stage de fin d'étude.

Enfin, nous tenons à remercier les deux rapporteurs anonymes ainsi que les rédacteurs de Norois dont les commentaires constructifs ont contribué à en améliorer sensiblement le contenu.

Cet article a été reçu le le 24 septembre 2007 et définitivement accepté le 26 mars 2008. 